\title{
DISTINGUISHEDNESS OF WEIGHTED FRÉCHET SPACES OF CONTINUOUS FUNCTIONS
}

\author{
by FRANÇOISE BASTIN
}

(Received 5th July 1990)

\begin{abstract}
In this paper, we prove that if $\mathscr{U}$ is an increasing sequence of strictly positive and continuous functions on a locally compact Hausdorf space $X$ such that $\vec{V} \simeq \nabla \cap C(X)$, then the Fréchet space $C \mathscr{U}(X)$ is distinguished if and only if it satisfies Heinrich's density condition, or equivalently, if and only if the sequence $\mathscr{U}$ satisfies condition $(H)$ (cf. e.g. '[1] for the introduction of $(H)$ ). As a consequence, the bidual $\lambda_{\infty}(A)$ of the distinguished Köthe echelon space $\lambda_{0}(A)$ is distinguished if and only if the space $\lambda_{1}(A)$ is distinguished. This gives counterexamples to a problem of Grothendieck in the context of Köthe echelon spaces.
\end{abstract}

1980 Mathematics subject classification (1985 Revision): 46E10, 46A45, 46A07.

\section{Introduction}

A locally convex space $E$ is distinguished if its strong dual is barrelled. All the Köthe echelon spaces $\lambda_{p}(A)$ of order $p=0$ or $1<p<\infty$ are known to be distinguished; in fact, for $1<p<\infty$, they are reflexive (cf. e.g. [4]), and the strong dual of $\lambda_{0}(A)$ is topologically isomorphic to the LB-space ind $_{n \rightarrow+\infty} l_{1}\left(a_{n}^{-1}\right), A=\left(a_{n}\right)_{n \in N}$. The situation for $p=1$ or $p=\infty$ is more complicated.

The distinguished spaces $\lambda_{1}(A)$ were characterized by K.-D. Bierstedt, J. Bonet and R. Meise (also see Vogt [6]): K.-D. Bierstadt and R. Meise [3] introduced the condition $(D)$ on a Köthe matrix $A$ and proved that $(D)$ implies $\lambda_{1}(A)$ distinguished. Then, K.-D. Bierstedt and J. Bonet [2] proved that in fact $(D)$ is also necessary for the distinguishedness of $\lambda_{1}(A)$.

Concerning the spaces $\lambda_{\infty}(A)$, which are the strong biduals of the corresponding spaces $\lambda_{0}(A)$, the problem of characterizing when $\lambda_{\infty}(A)$ is distinguished is related to the following question of Grothendieck: "Is the bidual of a distinguished Fréchet space also distinguished?" This question of Grothendieck has already been answered in the negative by J. Bonet, S. Dierolf and C. Fernandez [5]. These authors used Fréchet spaces of Moscatelli type to construct counterexamples. Moreover, they proved that this question is also related to the lifting of bounded sets: they show that if $E, F$ are Fréchet spaces such that $E \subset F \subset E^{\prime \prime}$ and if $F$ is distinguished, then $F / E$ is distinguished and its bounded sets are liftable (with closure). In our situation, this is a key point which allows us to forget about the dual of $\lambda_{\infty}(A)$, which is not a sequence space, and hence requires a new approach and other methods. 
In the present paper, we characterize the distinguished weighted Fréchet spaces of continuous functions on a locally compact Hausdorff space $X$ in terms of condition $(H)$ (cf. Notation). As a particular case, we obtain a characterization of the distinguished spaces $\lambda_{\infty}(A)$ : this space is distinguished if and only if $\lambda_{1}(A)$ is. Hence, concerning the preceding question of Grothendieck, we can say that every Köthe matrix $A$ which does not satisfy condition $(D)$ (or equivalently $(H)$, cf. Notation) gives a distinguished Fréchet space $\lambda_{0}(A)$ such that $\left(\lambda_{0}(A)\right)_{b b}^{\prime \prime} \simeq \lambda_{\infty}(A)$ is not distinguished.

\section{Notation}

Let $X$ denote a completely regular and Hausdorff space and $\mathscr{U}=\left(u_{m}\right)_{m \in N}$ denote a countable increasing system of strictly positive weights on $X$. We set

$$
v_{m}:=u_{m}^{-1}(m \in \mathbb{N}), \quad \mathscr{V}=\left(v_{m}\right)_{m \in \mathbb{N}}
$$

and

$$
\overline{\boldsymbol{V}}=\left\{\bar{v}: X \rightarrow\left[0,+\infty\left[; \sup _{\mathbf{q} \mathbf{x} \in \mathbf{X}}\left|\bar{v}(x) / v_{m}(x)\right|<+\infty, \forall m \in \mathbb{N}\right\}\right.\right.
$$

Then $C \mathscr{U}(X)$ denotes the linear space of all the continuous function $f$ on $X$ such that $p_{m}(f):=\sup _{x \in X} u_{m}(x)|f(x)|<+\infty \quad \forall m \in \mathbb{N}$ endowed with the locally convex topology defined by the semi-norms $p_{m}, m \in \mathbb{N}$. The notation $\lambda_{\infty}(A), A=\mathscr{U}$ is used in case $X$ is discrete. Further, $C \mathscr{U}_{0}(X)$ denotes the subspace of $C \mathscr{U}(X)$ consisting of all the continuous functions $f$ such that $u_{m} f$ converges to 0 at infinity for every $m \in \mathbb{N}$; in one case $X$ is discrete, $\lambda_{0}(A)$ is used instead of $C \mathscr{U}_{0}(X)$.

We will also use the following notation:

$Q$ for the quotient map $C \mathscr{U}(X) \rightarrow C \mathscr{U}(X) / C U_{0}(X)$,

$b_{m}$ for the neighbourhood $\left\{f \in \lambda_{\infty}(A): \sup _{x \in X}\left|u_{m}(x) f(x)\right| \leqq 1\right\}$ in $\lambda_{\infty}(A)$,

$D(X)$ for the space of all the continuous functions on $X$ with compact support $(D(X,[0,1])$ denotes then the set of the elements of $D(X)$ with values in $[0,1])$,

if $\bar{v} \in \bar{V}$, then $\bar{v}\left(l_{\infty}\right)_{1}$ is the set $\left\{f \in \lambda_{\infty}(A):|f(x)| \leqq \bar{v}(x), \forall x \in X\right\}$

$$
\left(=\left\{f \in \lambda_{\infty}(A): \exists g \in l_{\infty},|g(x)| \leqq 1 \forall x \in X: f=\bar{v} g\right\}\right) .
$$

Let us also recall the expressions of conditions $(D),(H),\left(H^{* *}\right)$ and $(N D)$, as well as the relations between them, cf. [1] (these expressions are given in terms of $\mathscr{V}$ or $\mathscr{U}$ ):

$$
\begin{aligned}
& \exists J=\left(X_{m}\right)_{m \in N}, \varnothing \neq X_{m} \subset X_{m+1} \forall m: \\
& (N, J) \forall n, \exists m(n): \inf _{x \in X_{n}} v_{k}(x) / v_{m(n)}(x)>0 \forall k \in \mathbb{N} \\
& (M, J) \forall n \text { and } Y,\left(Y \not \subset X_{m}, \forall m\right) \exists n^{\prime}: \inf _{y \in Y} v_{n}(y) / v_{n}(y)=0 ;
\end{aligned}
$$


(H) $\forall \lambda_{m}>0(m \in \mathbb{N}), \forall n \in \mathbb{N}, \exists \bar{v} \in \bar{V}$ and $M \in \mathbb{N}$ :

$$
\forall x \in X\left(\inf _{1 \leqq m \leqq M} \lambda_{m} v_{m}(x) \geqq v_{n}(x) \Rightarrow \bar{v}(x) \geqq v_{n}(x)\right) ;
$$

$\left(H^{* *}\right) \quad \forall \lambda_{m}>0(m \in \mathbb{N}) \exists \bar{v} \in \bar{V}: \forall n \in \mathbb{N}, \forall C>0, \exists M \in \mathbb{N}:$

$$
\forall x \in X\left(\inf _{1 \leqq m \leqq M} \lambda_{m} v_{m}(x) \geqq C v_{n}(x) \Rightarrow \bar{v}(x) \geqq C v_{n}(x)\right) ;
$$

(ND) $\exists n \in \mathbb{N}$ and a decreasing sequence $J_{k}(k \in \mathbb{N})$ of non void subsets of $X$ such that, $\forall k \geqq n:$

$$
\text { (i) } \inf _{x \in J_{k}} v_{k}(x) / v_{n}(x)>0 \text {; (ii) } \exists l(k)>k: \inf _{x \in J_{k}} v_{l(k)}(x) / v_{n}(x)=0 \text {. }
$$

It is known that $(D) \Leftrightarrow(H) \Leftrightarrow\left(H^{* *}\right) \Leftrightarrow \neg(N D)$.

\section{Main results}

As will be proved in Proposition 2, under some continuity assumption, the possibility of lifting the bounded sets of $C \mathscr{U}(X) / C \mathscr{U}_{0}(X)$ is equivalent to $(H)$ (or to $\neg(N D)$ ). To obtain this result, we need some more information about the sets $J_{k}(k \in \mathbb{N})$ appearing in $(N D)$ :

Lemma 1. If $\mathscr{V} \subset C(X)$, then in condition $(N D)$, we can assume that the sets $J_{k}$ 's are such that $\left(J_{k+1}\right)^{-} \subset J_{k}=\left(J_{k}\right)^{0}$ for every $k \in \mathbb{N}$, i.e.

(ND) $\exists n \in \mathbb{N}$ and a decreasing sequence $J_{k}(k \in \mathbb{N})$ of non void subsets of $X$ such that

$$
\begin{aligned}
\forall k \in \mathbb{N}: & \left(J_{k+1}\right)^{-} \subset J_{k}=\left(J_{k}\right)^{0}, \\
\forall k \geqq n: & \text { (i) } \inf _{x \in J_{k}} v_{k}(x) / v_{n}(x)>0, \\
& \text { (ii) } \exists l(k)>k: \inf _{x \in J_{k}} v_{l(k)}(x) / v_{n}(x)=0 .
\end{aligned}
$$

Proof. It is known that $(N D)$ is equivalent to $\neg(H)$. To obtain the result here, we just change the proof of $\urcorner(H) \Rightarrow(N D)$ of 1.2.7 of [1] slightly as follows.

As $(H)$ does not hold, there are $n \in \mathbb{N}$ and a sequence $\lambda_{m}>0(m \in \mathbb{N})$ such that

$$
\forall \bar{v} \in \bar{V}, \forall M \in \mathbb{N}, \exists x \in X: \inf _{1 \leqq m \leqq M} \lambda_{m} v_{m}(x) \geqq v_{n}(x) \text { and } \bar{v}(x)<v_{n}(x) .
$$

For every $k \in \mathbb{N}$, define

$$
J_{k}:=\left\{x \in X: \inf _{1 \leqq m \leqq k} \lambda_{m} v_{m}(x)>\left(1-2^{-k}\right) v_{n}(x)\right\}
$$


For every $k$, as (1) holds, the set $J_{k}$ is non void; and as the functions $v_{m}$ 's are continuous, we also have

$$
\left(J_{k+1}\right)^{-} \subset J_{k}=\left(J_{k}\right)^{0} \forall k \in \mathbb{N}
$$

Moreover, for every $k \in \mathbb{N}$, one gets

$$
\inf _{x \in J_{k}} \frac{v_{k}(x)}{v_{n}(x)} \geqq \frac{1-2^{-k}}{\lambda_{k}},
$$

hence (i) of $(N D)$ is satisfied.

So, to conclude, we just have to prove (ii). If (ii) is not satisfied, there is $k \geqq n$ such that

$$
\forall l>k, \delta_{l}:=\inf _{x \in J_{k}} \frac{v_{l}(x)}{v_{n}(x)}>0
$$

For $m>k$, let $\alpha_{m}:=\delta_{m}^{-1}$ and for $m=1, \ldots, k$, let $\alpha_{m}:=\lambda_{m}$. Then define $\bar{v}:=\inf _{m \in N} \alpha_{m} v_{m}$. Since (1) holds, there exists $x \in X$ such that

$$
\inf _{1 \leqq m \leqq k} \lambda_{m} v_{m}(x) \geqq v_{n}(x) \text { and } 2 \bar{v}<v_{n}(x)
$$

The first inequality implies $x \in J_{k}$. Moreover, by construction, $\forall y \in J_{k}$ we have

$$
\begin{array}{ll}
\lambda_{m} v_{m}(y)>\left(1-2^{-k}\right) v_{n}(y) \geqq 2^{-1} v_{n}(y) & \text { for } m=1, \ldots, k, \\
\delta_{m}^{-1} v_{m}(y) \geqq v_{n}(y) \geqq 2^{-1} v_{n}(y) & \text { for } m>k ;
\end{array}
$$

hence also $2 \bar{v}(y) \geqq v_{n}(y)$. But this contradicts $x \in J_{k}$ and $2 \bar{v}(x)<v_{n}(x)$.

Now we can prove the main result of this paper, i.e., the characterization of the lifting of the bounded sets of $C \mathscr{U}(X) / C \mathscr{U}_{0}(X)$ (with or without closure) in terms of condition $(H)$.

Proposition 2. Let $X$ be locally compact, $\mathscr{V} \subset C(X)$ and consider the following properties:

(1) $\mathscr{V}$ satisfies condition $(H)$ (or equivalently $\left(H^{* *}\right)$ );

(2) $\forall B$ bounded subset of $C \mathscr{U}(X) / C \mathscr{U}_{0}(X), \exists C$ bounded subset of $C \mathscr{U}(X)$ such that $B \subset Q(C)$;

(3) $\forall B$ bounded subset of $C \mathscr{U}(X) / C \mathscr{U}_{0}(X), \exists C$ bounded subset of $C \mathscr{U}(X)$ such that $B \subset(Q(C))^{-C \mathscr{Q}(X) / C \mathscr{Q}_{0}(X)}$.

Then $(1) \Rightarrow(3)$ and $(2) \Rightarrow(3)$. Moreover, if in addition we have $\bar{V} \simeq \bar{V} \cap C(X)$, then (3) $\Rightarrow(1)$ and $(1) \Rightarrow(2)$. 
Proof. Of course, $(2) \Rightarrow(3)$.

(1) $\Rightarrow$ (3). Given $B$, there is a sequence $\lambda_{m}>0(m \in \mathbb{N})$ such that

$$
B \subset \bigcap_{m \in N}\left(\left(\lambda_{m} b_{m} \cap C(X)\right)+C \mathscr{U}_{0}(X)\right) .
$$

Then condition $\left(H^{* *}\right)$ gives $\bar{v} \in \bar{V}$; we define

$$
\bar{u}:=\sup _{M \in N} \inf \left\{4 M \lambda_{M} \bar{v}, \lambda_{1} v_{1}, \ldots, \lambda_{M} v_{M}\right\}
$$

As $\bar{u}$ belongs also to $\bar{V}$, the set

$$
B^{\prime}:=2 \bar{u}\left(l_{\infty}\right)_{1} \cap C(X)
$$

is a bounded subset of $C \mathscr{U}(X)$. We claim that

$$
B \subset \bigcap_{n \in N}\left(C \mathscr{U}_{0}(X)+B^{\prime}+\left(\frac{1}{n} b_{n} \cap C(X)\right)\right) .
$$

Indeed, fix $n \in \mathbb{N}$ and take $f \in B$. Define the sets

$$
F:=\left\{x \in X:\left|u_{n}(x) f(x)\right| \leqq 1 / 2 n\right\} ; F^{\prime}:=\left\{x \in X:\left|u_{n}(x) f(x)\right| \geqq 1 / n\right\} .
$$

Then $F$ and $F^{\prime}$ are disjoint zero sets of continuous functions; so there is $g \in C(X,[0,1])$ such that $g=0$ on $F$ and $g=1$ on $F^{\prime}$. As we certainly have $f=g f+(1-g) f$ and $(1-g) f \in(1 / n) b_{n} \cap C(X)$, to conclude it remains to prove that $g f$ belongs to $B^{\prime}+C \mathscr{U}_{0}(X)$.

Using $\left(H^{* *}\right)$ with $n$ and $C=1 / 4 n$, we get $M=M(n)$ such that

$$
\forall x \in X\left(\inf _{1 \leqq m \leqq M} \lambda_{m} v_{m}(x) \geqq \frac{v_{n}(x)}{4 n} \Rightarrow \bar{v}(x) \geqq \frac{v_{n}(x)}{4 n}\right) .
$$

We can write (recall that $f$ belongs to $B$ )

$$
f=f^{(m)}+g^{(m)}, m=1, \ldots, M
$$

with $f^{(m)} \in \lambda_{m} b_{m} \cap C(X)$ and $g^{(m)} \in C \mathscr{U}_{0}(X)$ for every $m=1, \ldots, M$. Then there is a compact subset $K$ of $X$ such that

$$
\left|u_{n}(x) f(x)\right| \leqq \lambda_{m} u_{n}(x) v_{m}(x)+\frac{1}{4 n}
$$

for every $x \in X \backslash K$ and $1 \leqq m \leqq M$. It follows that every $x \in X \backslash(K \cup F)$ satisfies

$$
\frac{1}{4 n} v_{n}(x) \leqq \inf _{1 \leqq m \leqq M} \lambda_{m} v_{m}(x)
$$

hence also (use $\left(H^{* *}\right)$ )

$$
\frac{1}{4 n} v_{n}(x) \leqq \bar{v}(x)
$$


A look at the definition of $\bar{u}$ shows that the previous inequality implies

$$
\bar{u}(x) \geqq \inf \left\{4 M \lambda_{M} \bar{v}(x), \lambda_{1} v_{1}(x), \ldots, \lambda_{M} v_{M}(x)\right\}=\inf _{1 \leqq m \leqq M} \lambda_{m} v_{m}(x)
$$

for every $x \in X \backslash(K \cup F)$. Moreover, (2) and (3) implies also

$$
|f(x)| \leqq 2 \inf _{1 \leqq m \leqq M} \lambda_{m} v_{m}(x)(\leqq 2 \bar{u}(x))
$$

for every $x \in X \backslash(K \cup F)$. Taking now $\phi \in D(X,[0,1]), \phi=1$ on $K$, we get $g f=$ $\phi g f+(1-\phi) g f$, with $\phi g f \in D(X) \subset C \mathscr{U}_{0}(X)$. Finally, by construction and by (4), we obtain

$$
(1-\phi) g|f| \leqq 2 \bar{u} \text { on } X
$$

hence $(1-\phi) g f$ belongs to $B^{\prime}$ and we are done.

Now, assume that in addition, every element of $\vec{V}$ is dominated by a continuous element of $\bar{V}$.

$(3) \Rightarrow(1)$. We proceed by contradiction. If $(H)$ does not hold, condition $(N D)$ is satisfied and we can assume that it is satisfied with a decreasing sequence of non-void subsets $J_{k}(k \in \mathbb{N})$ verifying $\left(J_{k+1}\right)^{-} \subset J_{k}=\left(J_{k}\right)^{0}$ for every $k \in \mathbb{N}$. We can also suppose $n>1$.

For every $k \geqq n$, we set $\varepsilon_{k}:=\inf _{x \in J_{k}} u_{n}(x) / u_{k}(x)(>0, \operatorname{cf}(N D))$ and we define

$$
B:=\bigcap_{m \geqq n}\left(\left(\varepsilon_{m}^{-1} b_{m} \cap C(X)\right)+C \mathscr{U}_{0}(X)\right)
$$

which is a bounded subset of $C \mathscr{U}(X) / C \mathscr{U}_{0}(X)$.

As every bounded subset of $C \mathscr{U}(X)$ is contained in a set of the type $\bar{v}\left(l_{\infty}\right)_{1}(\bar{v} \in \bar{V})$, it remains to prove that $\forall \bar{v}=\inf _{m \in N} \rho_{m} v_{m},\left(\rho_{m}>0 \forall m\right)$, we have

$$
B \not C \mathscr{U}_{0}(X)+\left(\frac{1}{4} b_{n} \cap C(X)\right)+\left(\bar{v}\left(l_{\infty}\right)_{1} \cap C(X)\right)=: C^{\prime} .
$$

Indeed, let $\varepsilon_{m}:=1$ for $m=1, \ldots, n-1$ and take $\bar{v} \in \bar{V} \cap C(X)$ and a sequence $r_{m}>0 \forall m$ such that

$$
r_{m} \geqq \varepsilon_{m}^{-1} \forall m, \inf _{m \in N} r_{m} v_{m} \geqq \bar{v} \geqq 2 \quad \inf _{m \in N} \varepsilon_{m}^{-1} v_{m}
$$

Now, we use $(N D)$ and the fact that the $J_{k}$ 's are open to construct sequences $k(j) \in \mathbb{N}$, $x_{j} \in X$ and $V_{j} \subset X(j \in \mathbb{N})$ such that, $\forall j \in \mathbb{N}$

$$
k(1)=n, k(j)<k(j+1)
$$




$$
\begin{gathered}
V_{j}=\text { open neighbourhood of } x_{j} ; \\
V_{j} \subset J_{k(j)} \cap\left\{x: u_{n}(x) v_{k(j+1)}(x)<\inf \left\{\frac{1}{2 \rho_{k(j+1)}}, \frac{1}{2 r_{k(j+1)}}\right\}\right\}
\end{gathered}
$$

(hence $\quad V_{j} \subset J_{k(j)} \backslash\left(J_{k(j+1)}\right)^{-}, \quad \forall j \in \mathbb{N}$ and $V_{j} \cap V_{l}=\varnothing$ if $j \neq l$ ). Then, $\forall j \in \mathbb{N}$, let $f_{j} \in D(X,[0,1]), \operatorname{supp}\left(f_{j}\right) \subset V_{j}, f_{j}\left(x_{j}\right)=1$ and define

$$
f:=v_{n} \sum_{j=1}^{\infty} f_{j} .
$$

We claim that $f \in B \backslash C^{\prime}$. To prove this, we proceed in several steps.

(a) $f$ is continuous on $X$.

Indeed, take any $x \in X$.

If $x \in \bigcap_{j \in \mathbb{N}} J_{j}^{-}$, we obtain $\varepsilon_{m}^{-1} v_{m}(x) \geqq v_{n}(x) \forall m \in \mathbb{N}$, hence also

$$
\tilde{v}(x) \geqq 2 \inf _{m \in \mathrm{N}} \varepsilon_{m}^{-1} v_{m}(x)>v_{n}(x) .
$$

Since $v_{n}$ and $\tilde{v}$ are continuous, the set

$$
V:=\left\{y \in X: \tilde{v}(y)>v_{n}(y)\right\}
$$

is a neighbourhood of $x$. Moreover, for every $j \in \mathbb{N}$, we have

$$
V_{j} \subset\left\{y: 2 r_{k(j+1)} v_{k(j+1)}(y)<v_{n}(y)\right\} \subset\left\{y: 2 \tilde{v}(y)<v_{n}(y)\right\}
$$

hence $V_{j} \cap V=\varnothing$ and finally $f=0$ on $V$.

If there is $j_{0}$ such that $x \notin J_{j_{0}}^{-}$, then $V=X \backslash\left(J_{j_{0}}\right)^{-}$is an open neighbourhood of $x$ which meets only finitely many $V_{j}$ 's (because $V_{l} \subset J_{k(l)} \forall l$ and $J_{k(l)} \subset J_{j_{0}}$ for $l \geqq j_{0}$ ), hence $\left.f\right|_{V}$ is a finite sum of continuous functions.

(b) $f$ belongs to $C \mathscr{U}(X)$.

Indeed, fix $m \in \mathbb{N}, m \geqq n$. We have

$$
\begin{aligned}
\sup _{x \in X} u_{m}(x)|f(x)| & =\sup _{j \in \mathbb{N}, x \in V_{j}} \frac{u_{m}(x)}{u_{n}(x)} f_{j}(x) \\
& \leqq \sup \left\{\sup _{x \in \bigcup y_{j=1} 1^{1} \operatorname{supp}\left(f_{j}\right)} \frac{u_{m}(x)}{u_{n}(x)}, \varepsilon_{m}^{-1}\right\}
\end{aligned}
$$

and hence the required conclusion.

(c) $f$ belongs to $B=\bigcap_{m \geqq n}\left(\left(\varepsilon_{m}^{-1} b_{m} \cap C(X)\right)+C \mathscr{U}_{0}(X)\right)$.

Indeed, fix $m \in \mathbb{N}, m \geqq n$. Then 


$$
f=f^{(1, m)}+f^{(2, m)}
$$

with

$$
f^{(1, m)}=v_{n} \sum_{j=1}^{m-1} f_{j} ; \quad f^{(2, m)}=v_{n} \sum_{j=m}^{+\infty} f_{j}
$$

and

$$
f^{(1, m)} \in D(X) \subset C \mathscr{U}_{0}(X) ; \quad f^{(2, m)} \in \varepsilon_{m}^{-1} b_{m} \cap C(X)
$$

(d) Assume that there are $g \in(1 / 4) b_{n} \cap C(X), h \in\left(l_{\infty}\right)_{1}$ and $w \in C \mathscr{U}_{0}(X)$ such that $f=g+\bar{v} h+w$. Then, for every $j \in \mathbb{N}$, we have

$$
\begin{aligned}
1=u_{n}\left(x_{j}\right) v_{n}\left(x_{j}\right)=u_{n}\left(x_{j}\right) f\left(x_{j}\right) & \leqq \frac{1}{4}+u_{n}\left(x_{j}\right)\left|w\left(x_{j}\right)\right|+\rho_{k(j+1)} v_{k(j+1)}\left(x_{j}\right) u_{n}\left(x_{j}\right) \\
& <\frac{1}{4}+u_{n}\left(x_{j}\right)\left|w\left(x_{j}\right)\right|+\frac{1}{2} .
\end{aligned}
$$

As $w$ belongs to $C\left(u_{n}\right)_{0}(X)$, to conclude, it suffices now to prove that the set $\left\{x_{j}: j \in \mathbb{N}\right\}$ is not relatively compact.

Indeed, if it was compact, we could find $x_{0} \in \bigcap_{N \in N}\left\{x_{j}: j \geqq N\right\}^{-}$. But for every $N \in \mathbb{N}$ and $j \geqq N$, we have $x_{j} \in V_{j} \subset J_{k(j)} \subset J_{k(N)} \subset J_{N}$, which implies $\varepsilon_{N}^{-1} v_{N}\left(x_{j}\right) \geqq v_{n}\left(x_{j}\right)$ and finally the inclusion

$$
\left\{x_{j}: j \geqq N\right\}^{-} \subset\left\{x \in X: \varepsilon_{N}^{-1} v_{N}(x) \geqq v_{n}(x)\right\}
$$

This implies that $x_{0}$ satisfies $\inf _{m \in N} \varepsilon_{m}^{-1} v_{m}\left(x_{0}\right) \geqq v_{n}\left(x_{0}\right)$ hence also $\bar{v}\left(x_{0}\right)>v_{n}\left(x_{0}\right)$. As in the case (a) above, $V=\left\{x \in X: \tilde{v}(x)>v_{n}(x)\right\}$ is then a neighbourhood of $x_{0}$ and it follows that there exists $M$ such that $\tilde{v}\left(x_{M}\right)>v_{n}\left(x_{M}\right)$. But this implies

$$
r_{k(M+1)} v_{k(M+1)}\left(x_{M}\right)>v_{n}\left(x_{M}\right)
$$

which is a contradiction because

$$
x_{M} \in V_{M} \subset\left\{x: \frac{u_{n}(x)}{u_{k(M+1)}(x)}<\frac{1}{2 r_{k(M+1)}}\right\}
$$

$(1) \Rightarrow(2)$. We improve the proof of $(1) \Rightarrow(3)$ in the case $\bar{V}$ that satisfies the continuous domination property (i.e. $\bar{V} \simeq \bar{V} \cap C(X)$ ).

As $B$ is bounded in $C \mathscr{U}(X) / C \mathscr{U}_{0}(X)$, there is a sequence $\lambda_{m}>0(m \in \mathbb{N})$ such that

$$
B \subset \bigcap_{m \in N}\left(\left(\lambda_{m} b_{m} \cap C(X)\right)+C \mathscr{U}_{0}(X)\right)
$$

Using $\left(H^{* *}\right)$ (equivalent to $(H)$ ), we get $\bar{v} \in \bar{V}$ such that $\forall n \in \mathbb{N}, \exists M(n) \geqq n$ : 


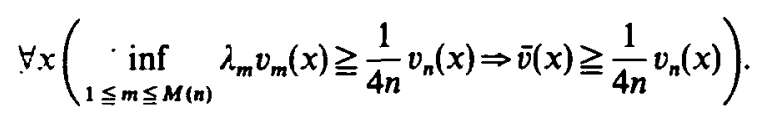

We define

$$
\bar{u}^{\prime}:=\sup _{m \in N} \inf \left\{4 M \lambda_{M} \bar{v}, \lambda_{1} v_{1}, \ldots, \lambda_{M} v_{m}\right\}
$$

We have $\bar{u}^{\prime} \in \bar{V}$. Let $\tilde{u}$ be a strictly positive (the condition $(H)$ implies the existence of a strictly positive element of $\bar{V}$ ) and continuous element of $\bar{V}$ such that $\tilde{u} \geqq 2 \inf _{k \in N} \lambda_{k} v_{k}$ and $\bar{u} \geqq \bar{u}^{\prime}$. We claim that

$$
B^{\prime}:=\bigcap_{m \in N}\left(\lambda_{m} b_{m} \cap C(X)+C \mathscr{U}_{0}(X)\right) \subset 8 \tilde{u}\left(l_{\infty}\right)_{1}+C \mathscr{U}_{0}(X) .
$$

Indeed, let $f \in B^{\prime}$. For every $m \in \mathbb{N}$, there are $f^{(m)} \in \lambda_{m} b_{m} \cap C(X)$ and $g^{(m)} \in C \mathscr{U}_{0}(X)$ such that $f=f^{(m)}+g^{(m)}$. Hence, for every $n \in \mathbb{N}$, there exists a compact subset $K_{n} \subset X$ such that

$$
u_{n}(x)\left|g^{(k)}(x)\right| \leqq \frac{1}{4 n} ; \forall k=1, \ldots, M(n) ; \forall x \in X \backslash K_{n} .
$$

We set $K_{0}:=\varnothing$; moreover, in the previous construction (this construction is possible except if $X$ is compact. But then, the property is of course true and we have nothing to prove), we can assume that $\varnothing \neq K_{n} \subsetneq\left(K_{n+1}\right)^{0}, \forall n \in \mathbb{N}$.

We proceed again in several steps.

(a) Let $\phi_{1}^{\prime} \in D(X,[0,1])$ be such that $\phi_{1}^{\prime}=1$ on $K_{1}$, supp $\left(\phi_{1}\right) \subset\left(K_{2}\right)^{0}$ and, for every $n \geqq 2$, let $\phi_{n}^{\prime} \in D(X,[0,1])$ be such that $\phi_{n}^{\prime}=1$ on $K_{n} \backslash\left(K_{n-1}\right)^{0}, \operatorname{supp}\left(\phi_{n}\right) \subset\left(K_{n+1}\right)^{0} \backslash K_{n-2}$. Moreover, as the sets $F:=\{x \in X:|f(x)| \leqq \tilde{u}(x)\}$ and $G:=\{x \in X:|f(x)| \geqq 2 \tilde{u}(x)\}$ are disjoint zero-sets of continuous functions, there is $h \in C(X,[0,1])$ satisfying $h=0$ on $F, h=1$ on $G$.

We show that $1-h, h \phi_{n}^{\prime}(n \in \mathbb{N})$ form a locally finite family $\mathscr{F}$ of continuous functions on $X$ such that $\phi^{\prime}(x):=1-h(x)+\sum_{n=1}^{+\infty} h(x) \phi_{n}^{\prime}(x)>0, \forall x \in X$.

Indeed, for every $x \in \bigcap_{n \in \mathbb{N}}\left(X \backslash K_{n}\right)$, we have $g^{(k)}(x)=0 \forall k \in \mathbb{N}$ (cf. (6)), hence $|f(x)| \leqq$ $\lambda_{k} v_{k}(x) \forall k \in \mathbb{N}$ and finally $|f(x)| \leqq \inf _{k \in \mathbb{N}} \lambda_{k} v_{k}(x)<\tilde{u}(x)$; it follows that $F$ is a neighbourhood of $\bigcap_{n \in N}\left(X \backslash K_{n}\right)$. Then, as $h=0$ on $F$, the family $\mathscr{F}$ is locally finite on $\bigcap_{n \in \mathbb{N}}\left(X \backslash K_{n}\right)$, and, by construction of the functions $\phi_{n}^{\prime}$, it is also locally finite on $\bigcap_{n \in N} K_{n}$.

To prove that $\phi^{\prime}(x)>0 \forall x \in X$, is suffices to remark that

$$
h(x) \neq 0 \Rightarrow x \in \bigcup_{m \in N} K_{n} \Rightarrow\left\{\begin{array}{l}
3 \geqq \sum_{n=1}^{+\infty} \phi_{n}^{\prime}(x) \geqq 1 \\
\phi^{\prime}(x)=1+h(x)\left(-1+\sum_{n=1}^{+\infty} \phi_{n}^{\prime}(x)\right)>0 .
\end{array}\right.
$$

We set 


$$
\phi_{0}:=\frac{1-h}{\phi^{\prime}}, \quad \phi_{n}:=\frac{h \phi_{n}^{\prime}}{\phi^{\prime}}(n \in \mathbb{N})
$$

(b) For every $n \in \mathbb{N}$, let us define

$$
F_{1}^{(n)}:=\left\{x \in X: u_{n}(x)|f(x)| \leqq 1 / 2 n\right\} ; \quad F_{2}^{(n)}:=\left\{x \in X: u_{n}(x)|f(x)| \leqq 1 / n\right\}
$$

and take $h^{(n)} \in C(X,[0,1])$ such that $h^{(n)}=0$ on $F_{1}^{(n)}, h^{(n)}=1$ on $F_{2}^{(n)}$. For every $x \in X \backslash\left(K_{n} \cup F_{1}^{(n)}\right)$, we have (cf. the decompositions $f=f^{(m)}+g^{(m)}$ of $f$ )

$$
\frac{1}{2 n}<u_{n}(x)|f(x)| \leqq u_{n}(x) \inf _{1 \leqq k \leqq M(n)} \lambda_{k} v_{k}(x)+\frac{1}{4 n},
$$

hence

$$
\frac{1}{4 n} v_{n}(x)<\inf _{1 \leqq k \leqq M(n)} \lambda_{k} v_{k}(x)
$$

and (from (5))

$$
\frac{1}{4 n} v_{n}(x) \leqq \bar{v}(x)
$$

It follows that

$$
\inf _{1 \leqq k \leqq n} \lambda_{k} v_{k}(x) \leqq \bar{u}^{\prime}(x) \leqq \tilde{u}(x)
$$

and that

$$
\begin{aligned}
|f(x)| & \leqq \inf _{1 \leqq k \leqq M(n)} \lambda_{k} v_{k}(x)+\frac{v_{n}(x)}{4 n} \\
& \leqq 2 \inf _{1 \leqq k \leqq M(n)} \lambda_{k} v_{k}(x) \\
& \leqq 2 \tilde{u}(x) .
\end{aligned}
$$

(c) Now, $f$ can be decomposed as follows:

$$
f=f \phi_{0}+f\left(\phi_{1}+\phi_{2}\right)+\sum_{n=3}^{+\infty} f \phi_{n} h^{(n-2)}+\sum_{n=3}^{+\infty} f \phi_{n}\left(1-h^{(n-2)}\right)
$$

For every $n \geqq 3$, we have

$$
f \phi_{n}(x) h^{(n-2)}(x) \neq 0 \Rightarrow\left\{\begin{array}{l}
x \notin F_{1}^{(n-2)} \\
x \in \operatorname{supp}\left(\phi_{n}\right) \subset\left(K_{n+1}\right)^{0} \backslash K_{n-2} \subset X \backslash K_{n-2},
\end{array}\right.
$$


hence $|f(x)| \leqq 2 \tilde{u}(x)$ and finally

$$
\left|\sum_{n=3}^{+\infty} f(x) \phi_{n}(x) h^{(n-2)}(x)\right| \leqq 6 \tilde{u}(x) \quad \forall x \in X
$$

Next, let us verify that $\sum_{n=3}^{+\infty} f \phi_{n}\left(1-h^{(n-2)}\right)$ belongs to $C \mathscr{U}_{0}(X)$. First, this function clearly belongs to $C \mathscr{U}(X)$. Now fix $N \in \mathbb{N}$ and $\varepsilon>0$. For every $n \geqq 3$, we have

$$
f \phi_{n}(x)\left(1-h^{(n-2)}(x)\right) \neq 0 \Rightarrow x \in X \backslash F_{2}^{(n-2)} \Rightarrow u_{n-2}(x)|f(x)| \leqq \frac{1}{n-2} .
$$

Hence, if $N^{\prime} \in \mathbb{N}$ is such that $N^{\prime} \geqq \sup \left\{N+2,3 \varepsilon^{-1}+2\right\}$, for every $x \notin K_{N^{\prime}}$, we get

$$
\begin{aligned}
u_{N}(x)\left|\sum_{n=3}^{+\infty} f \phi_{n}(x)\left(1-h^{(n-2)}(x)\right)\right| & =u_{N}(x)\left|\sum_{n=N^{\prime}}^{+\infty} f \phi_{n}(x)\left(1-h^{(n-2)}(x)\right)\right| \\
& \leqq \sum_{n=N^{\prime}}^{+\infty}|f(x)| \phi_{n}(x) u_{n-2}(x)\left(1-h^{(n-2)}(x)\right) \\
& \leqq \frac{3}{N^{\prime}-2} \leqq \varepsilon
\end{aligned}
$$

Finally, as $\phi_{0}=0$ on $G=\{x \in X:|f(x)| \geqq 2 \tilde{u}(x)\}$, we also have $|f(x)| \phi_{0}(x) \leqq 2 \tilde{u}(x)$, $\forall x \in X$.

Hence the conclusion: $f$ belongs to the set $8 \tilde{u}\left(l_{\infty}\right)_{1}+C \mathscr{U}_{0}(X)$.

Let us now recall Lemma 1 of [5] which shows how distinguishedness and lifting of bounded sets are connected.

Lemma 3 ([5]). Let $E, F$ be Fréchet spaces such that $E \subset F \subset E^{\prime \prime}$, and let $q: F \rightarrow F / E$ denote the quotient map. Assume that $F$ is distinguished. Then

(i) $F / E$ is distinguished, and

(ii) $\forall$ bounded subset $B$ of $F / E, \exists A$ bounded subset of $F$ such that $B \subset(q(A))^{-}$.

Proposition 2 and the lemma recalled above lead now to the following result.

Theorem 4. Let $X$ be locally compact, $\mathscr{V} \subset C(X)$ and $\bar{V} \simeq \bar{V} \cap C(X)$. Then the following properties are equivalent:

(1) $\operatorname{CO}(X)$ is distinguished;

(2) $C \mathscr{U}(X)\left(\right.$ resp. CUU $\mathscr{U}_{0}(X)$ ) satisfies $S$. Heinrich's density condition;

(3) $\mathscr{V}$ satisfies $(H)$; 
(4) $\forall B$ bounded subset of $C \mathscr{U}(X) / C \mathscr{U}_{0}(X), 3 C$ bounded subset of $C \mathscr{U}(X)$ such that $B \subset Q(C)$;

(5) $\forall B$ bounded subset of $C \mathscr{U}(X) / C \mathscr{U}_{0}(X), \exists C$ bounded subset of $C \mathscr{U}(X)$ such that

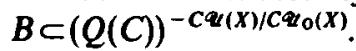

Proof. From [1], we know that (2) and (3) are equivalent (this result is valid without the assumption $\bar{V} \simeq \bar{V} \cap C(X))$.

The equivalence between (3), (4) and (5) is proved in the preceding proposition.

As $E=C \mathscr{U}_{0}(X)$ and $F=C \mathscr{U}(X)$ are Fréchet spaces satisfying $E \subset F \subset E^{\prime \prime}$, we can apply Lemma 1 of [5] and we get $(1) \Rightarrow(5)$.

As S. Heinrich's density condition for Fréchet spaces implies distinguishedness, the proof is complete.

Corollary 5. Let $A=\left(a_{n}\right)_{n \in N}$ be a Köthe matrix on a discrete space $X$ and let $q$ denote the quotient map $\lambda_{\infty}(A) \rightarrow \lambda_{\infty}(A) / \lambda_{0}(A)$. Then the following properties are equivalent:

(1) $\lambda_{\infty}(A)$ is distinguished;

(2) $\lambda_{1}(A)$ is distinguished;

(3) $\lambda_{\infty}(A)$ (resp. $\lambda_{1}(A)$ ) satisfies $S$. Heinrich's density condition;

(4) $\forall B$ bounded subset of $\lambda_{\infty}(A) / \lambda_{0}(A), \exists C$ bounded subset of $\lambda_{\infty}(A)$ such that $B \subset q(C)$;

(5) $\forall$ Bboundedsubset of $\lambda_{\infty}(A) / \lambda_{0}(A), \exists$ Cboundedsubset of $\lambda_{\infty}(A)$ such that $B \subset(q(C))^{-\lambda_{\infty}(A) / \lambda_{0}(A)}$.

Remark. Completely independently from this paper, E. Shalück (Universität-GHPaderborn) obtained results about the distinguishedness of weighted spaces $C V_{0}(X)$. He proved that if $V$ is an increasing sequence of strictly positive and continuous functions on a locally compact Hausdorff space $X$ such that every lower semi-continuous $v: X \rightarrow\left[0,+\infty\left[\cup\{\infty\}\right.\right.$ satisfying $\sup _{x \in X} v_{n}(x) / v(x)<\infty \forall n \in \mathbb{N}$ is dominated by a continuous function of the same type, then $C V_{0}(X)$ is distinguished.

Acknowledgement. We are particularly grateful to S. Dierolf whose stimulating discussions, suggestions and encouragement led us to write this paper.

\section{REFERENCES}

1. F. Bastin, Weighted spaces of continuous functions, Bull. Soc. Roy. Sci. Liège 1 (1990), $1-81$.

2. K.-D. Bierstedt and J. Bonet, Stefan Heinrich's density condtion for Fréchet spaces and the characterization of the distinguished Köthe echelon spaces, Math. Nachr. 135 (1988), 149-180.

3. K.-D. Bierstedt and R. Meise, Distinguished echelon spaces and the projective description of the weighted inductive limits of type $\mathscr{V} C(X)$, in Aspects of Mathematics and its Applications (Elsevier Science Publ. B.V. North-Holland Math. Library, 1986).

4. K.-D. Bierstedt, R. Meise and W. Summers Köthe sets and Köthe sequence space, in Functional Analysis, Holomorphy and Approximation Theory (North-Holland Math. Studies 71 , 1982), 27-91. 
5. J. Bonet, S. Dierolf and C. Fernandez, The bidual of a distinguished Fréchet space need not be distinguished (1990), preprint.

6. D. Vogt, Distinguished Köthe spaces, Math. Z. 202 (1989), 143-146.

UNIVERSITE DE LIÈGE

Institut de Mathematique

Avenue des Tilleuls, 15

4000 Liege, Belgium 